\title{
Stability of Boolean and continuous dynamics
}

\author{
Fakhteh Ghanbarnejad and Konstantin Klemm* \\ Bioinformatics Group, Institute for Computer Science, \\ University of Leipzig, Härtelstraße 16-18, D-04107 Leipzig, Germany
}

\begin{abstract}
Regulatory dynamics in biology is often described by continuous rate equations for continuously varying chemical concentrations. Binary discretization of state space and time leads to Boolean dynamics. In the latter, the dynamics has been called unstable if flip perturbations lead to damage spreading. Here we find that this stability classification strongly differs from the stability properties of the original continuous dynamics under small perturbations of the state vector. In particular, random networks of nodes with large sensitivity yield stable dynamics under small perturbations.
\end{abstract}

PACS numbers: 89.75.Hc,05.45.-a,87.10.-e,45.05.+x

The functioning of organisms on the molecular level is a research topic of increasing attention. Survival and reproduction requires an autonomous regulation of chemical concentrations in the living cell. Modeling such regulatory dynamics, various mathematical approaches have been studied, from discrete to continuous methods, from deterministic to stochastic techniques, from static to dynamical models, from detailed to coarse grained perspectives [1], see ref. [2] for an overview.

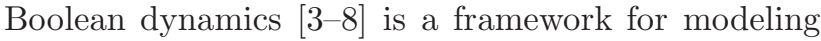
regulatory systems, especially for precise sequence control as observed in morphogenesis [9] and cell cycle dynamics [10] but also in the regulation of the metabolism [11]. Using binary (on/off) concentrations as an idealization, Boolean dynamics directly implements the logical skeleton of regulation. Values of system parameters such as binding constants, production and degradation rates etc. are not needed. This abstraction simplifies computation and analytical treatment. Boolean networks have been extracted directly from the literature [6, 12] of known biochemical interactions or obtained by discretization of differential equation models [13]. Known state sequences and responses of several systems have been faithfully reproduced by the discrete models [9, 10].

Despite these benefits, modelers do not employ Boolean dynamics as widely as ordinary or delay differential equations. The latter are embedded in an established framework for state-continuous dynamical systems [14] which itself builds on the mathematical foundations of linear algebra and infinitesimal calculus. In particular, the definition of stability of a solution under small perturbations is based on the consideration of infinitesimally small neighborhoods in state space. Stability checks for solutions of the dynamical equations are a salient part of mathematical modeling. Unstable solutions are not expected to be observed in a real-world system.

In the state-discrete Boolean dynamics, large perturbations are normally implemented as a flip, where the state of a single Boolean variable is inverted. Then the evolution of the damage is tracked. The damage is the

*Electronic address: fakhteh,klemm@bioinf.uni-leipzig.de difference between the state of the perturbed and the unperturbed system. The return map of the expected size of the damage is known as Derrida plot [15]. Numerous studies have elucidated the effect of flip perturbations on regulatory dynamics with Boolean states [16 21]. When asking if a gene-regulatory system reproduces a prescribed trajectory despite noise, large perturbations are to be considered in the case of low copy numbers of regulatory molecules and bursty stochastic response 22]. Small perturbations, however, are more appropriate when modeling systems with large copy numbers and an integrative response to filter out bursts, see e.g. [23].

Here we find that the clear distinction between the two types of perturbations is crucial. In a continuous system, stability or instability under small perturbations is not indicative of the effect of flip perturbations. Likewise, probing a Boolean system with flip perturbations does not necessarily provide information about the stability of the continuous counterpart under small perturbations.

An $n$-dimensional Boolean map $f:\{0,1\}^{n} \rightarrow\{0,1\}^{n}$ gives rise to a time-discrete dynamics

$$
x(t+1)=f(x(t))
$$

with $x=\left(x_{1}, \ldots, x_{n}\right)$ being a Boolean state vector (bit string) of $n$ entries. Such a map is equivalent to a Boolean network. When $f$ is pictured as a network, a node corresponds to a coordinate $i$ of the Boolean state vector and a directed edge $j \rightarrow i$ (from node $j$ to node $i$ ) is present if the Boolean function $f_{i}$ explicitly depends on the $j$-th coordinate.

Let us now define a continuous dynamics whose discretization readily leads to the Boolean map in Eq. (1). Taking values $y_{i}(t) \in[0,1], i \in\{1, \ldots, n\}, t \in \mathbb{R}$, the states evolve according to the delay differential equation

$$
\dot{y}_{i}(t+1)=\alpha \operatorname{sgn}\left(\tilde{f}(y(t))-y_{i}(t+1)\right)
$$

with $\alpha$ an inverse time constant. For large $\alpha$, this is essentially Boolean dynamics with fast but continuous switching between the saturation values. The simplest choice is $\tilde{f}=f \circ \Theta$ with $\Theta$ the component-wise step function, $\Theta_{i}(y)=1$ if $y_{i} \geq 1 / 2$ and $\Theta_{i}(y)=0$ otherwise. This choice of continuous dynamics is in close correspondence with the discrete dynamics in the following sense. 
Suppose $x(0), x(1), x(2), \ldots$ is a solution of Eq. (11). Let $y(t)$ be a solution of Eq. (2) such that there is a time interval $\left[t_{1}, t_{2}\right]$ with $y(s)=x(0)$ for all $s \in\left[t_{1}, t_{2}\right]$. Then for all future times $t \in \mathbb{N}$ and all $s \in\left[t_{1}, t_{2}\right]$

$$
x(t)=y(\beta t+s)
$$

with $\beta=1+1 /(2 \alpha)$. The closest resemblance between Boolean and continuous dynamics is obtained when choosing the same initial condition, that is $y(s)=$ $x(0)$ for all $s \in[-1,0]$. Similar correspondence between Boolean maps and ordinary differential equations has been studied earlier neglecting transmission delay [24] or implementing more complicated differential equations [25 28] compared to Equation (2).

Perturbations. - Given a map $f$, the evolution of states is uniquely determined by Eq. (2) by an initial condition $y(t)$ on a time interval of unit length, here taken as $[-1,0]=: I$. We restrict ourselves to initial conditions that do not vary on $I, y(t)=y(0)$ for all $t \in I$. An initial condition with a small perturbation is generated as

$$
y_{i}^{\prime}(t):=\epsilon_{i}\left(1-y_{i}(t)\right)+\left(1-\epsilon_{i}\right) y_{i}(t)
$$

for $t \in I$. The perturbation amplitudes are arbitrary numbers $\left.\epsilon_{i} \in\right] 0,1 / 2[$. An initial condition with a flip perturbation is generated as

$$
y_{i}^{!}(t):=\left\{\begin{aligned}
1-y_{i}(t) & \text { if } i=l \\
y_{i}(t) & \text { otherwise }
\end{aligned}\right.
$$

for $t \in I$ and an arbitrary node $l \in\{1, \ldots, n\}$. Note that the total amplitude $\sum_{i} \epsilon_{i}$ of a small perturbation may exceed the unit amplitude of a flip perturbation. A small perturbation produces small deviations from the original state potentially at each node. A flip perturbation concentrates a maximal deviation at a single node.

We say that the system heals from the perturbation if the dynamics from perturbed and unperturbed initial condition eventually become the same except for an arbitrary time lag. Formally, healing from a small perturbation means that there are $t_{0}>0$ and $\tau>-t_{0}$ such that

$$
y(t)=y^{\prime}(t+\tau)
$$

for all $t \geq t_{0}$. Healing from a flip perturbation means

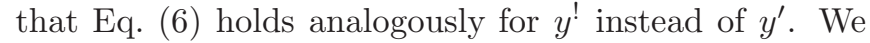
define the heal time $t_{\text {heal }}$ as the smallest time $t_{0}$ for which this holds.

Fixed points and bistable circuits. - Let us first consider a fixed point as the simplest dynamical behaviour. A fixed point of the continuous dynamics is a state vector $y^{*}$ such that constant $y(t)=y^{*}$ is a solution of Eq. (2). This in turn means that the time derivative vanishes at all times, equivalent to $y^{*}=f\left(y^{*}\right)$. The fixed points of the continuous dynamics are exactly the fixed points of the discrete map $f$. A small perturbation to a fixed point $y^{*}$ always heals, because values after applying the treshold $\Theta$ remain unchanged, $\tilde{f}\left(y^{\prime}(t)\right)=y^{*}$ for all $t \in I$. All
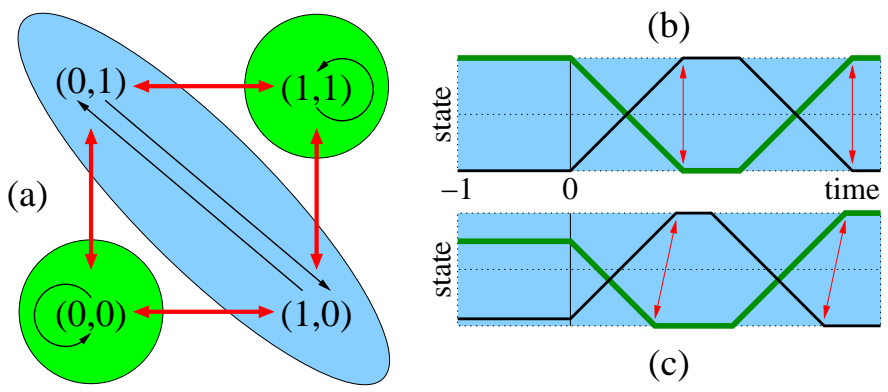

(c)

FIG. 1: (color online). Dynamics of two mutually activating nodes. (a) State space of the Boolean system described by Eq. (7). Thin arrows indicate the mapping $f$ of states by the dynamics, thick bidirectional arrows stand for flip perturbations. Indicated by shaded areas, the system has three dynamical modes (attractors): two fixed points $(0,0)$ and $(1,1)$ and a cycle of length 2 involving the states $(0,1)$ and $(1,0)$. (b) Time evolution of the corresponding continuous system in Equation (2) with initial condition $x_{1}(0)=1$ (thick curve) and $x_{2}(0)=0$ (thin curve). The two nodes switch in a synchronous mode as indicated by vertical double arrows akin to the Boolean state sequence $(0,1),(1,0),(0,1), \ldots$ (c) Time evolution from perturbed initial condition, $x_{1}(0)<1$, $x_{2}(0)>0$. The perturbation translates into a phase lag in switching that does not heal out.

fixed points are stable under small perturbations. However, a flip perturbation to a fixed point does not always heal. The bistable switch is an example. Consider a twodimensional map $f$ with $f\left(x_{1}, x_{2}\right)=\left(x_{2}, x_{1}\right)$. It gives rise to the dynamics

$$
x_{1}(t+1)=x_{2}(t) \quad x_{2}(t+1)=x_{1}(t)
$$

with fixed points $(0,0)$ and $(1,1)$. After perturbing a fixed point by flipping one node's state, the system does not return to the fixed point. It remains in the set of the state vectors $(0,1)$ and $(1,0)$ constituting a limit cycle, cf. Figure 1(a). The stability of the fixed points is not obtained when probing the dynamics with flip perturbations. The bistable switch constitutes a first simple example of systems with different stability properties under flip and small perturbations.

In the continuous counterpart of the alternating Boolean state $(0,1)$ and $(1,0)$, small perturbations do not heal, see Figure 1 $(b, c)$. The effect of a small perturbation is to induce a phase lag in the oscillation, being discussed in earlier work [25, 29 31].

Stability in random networks. - We now compare the effects of the two types of perturbations on dynamics in randomly generated networks. An ensemble of random Boolean networks (RBN) [5] is defined by the number of nodes $n$, the number of inputs $K$ of each node, and the probability distribution of Boolean functions $\pi(f)$. The latter is taken as a maximum entropy ensemble $\pi_{\lambda}(f) \propto \exp (\lambda s(f))$ under a given average sensitivity $\langle s\rangle$. The sensitivity $s(f)$ of a Boolean function $f$ is the number of flips at one of the $K$ inputs that lead to a change of the output value, averaged over all input vectors [32]. 


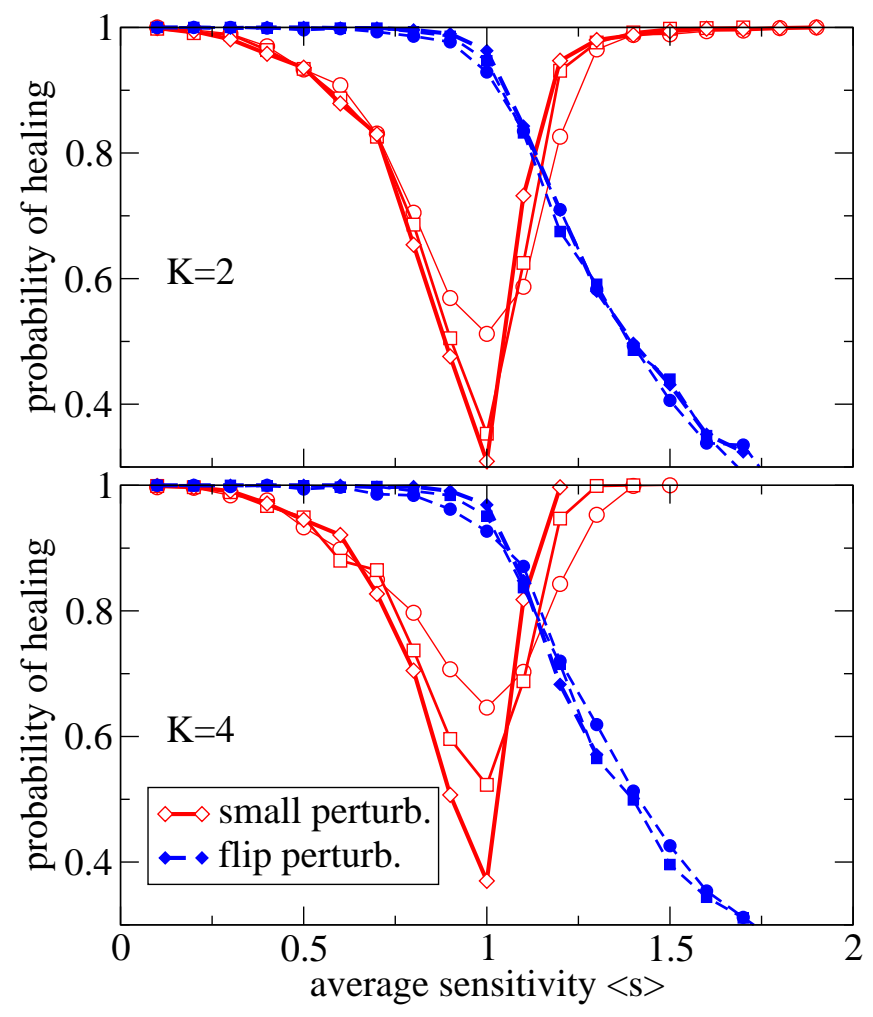

FIG. 2: (color online). Stability of dynamics in random networks under perturbation by spin flip (dashed) and under continuous perturbation (solid lines) in random networks with $K=2$ and $K=4$ inputs per node. Symbols distinguish system size $n=300(\circ), 1000(\square)$ and $3000(\diamond)$. Each data point gives the relative frequency of healed out perturbations on a set of $10^{4}$ independent random realizations of network, initial condition and perturbation. Each amplitude $\epsilon_{i}$ of a small perturbation is drawn independently from the uniform distribution on an interval $[0 ; r]$ with $0<r<0.5$. The results are independent of the choice of $r$. As a general invariance of the dynamics of Equation (2) with $\tilde{f}=f \circ \Theta$, the qualitative effect (healing or spreading) of a small perturbation is not altered when the amplitude vector is multiplied with a positive scalar keeping each amplitude $\epsilon_{i}<0.5$.

The resulting value $s(f)$ lies in the range from zero (for a constant function $f$ ) to $K$, obtained for a parity function where for all input vectors, a flip of a single input state flips the output. For RBN, where the $K$ inputs of each node are drawn randomly and independently from the set of $n$ nodes, the average sensitivity $\langle s\rangle$ is the crucial parameter determining the system's response to flip perturbations [32]. In the limit $n \rightarrow \infty$, these perturbations heal in ensembles with $\langle s\rangle<1$; they spread when $\langle s\rangle>1$. This change of behaviour in dependence of $\langle s\rangle$ is reproduced in Figure 2 (dashed lines) for varying $K$ and $n$.

As our main result, we show in Fig. 2 that the $\langle s\rangle$ dependence of the healing probability under flip perturbations is qualitatively different from that under small perturbations. Only in the so-called critical region of

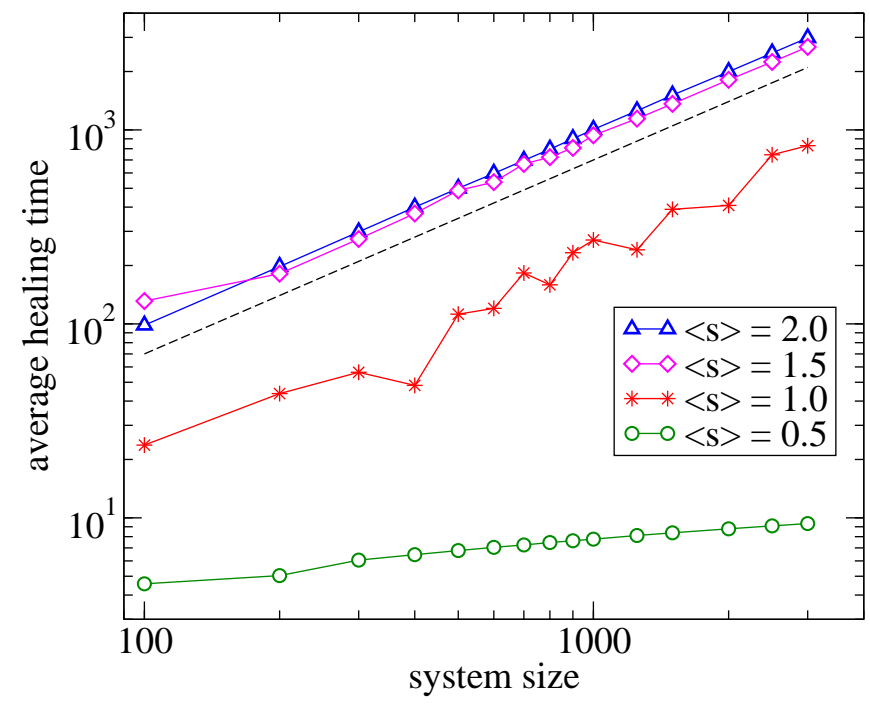

FIG. 3: (color online). The average time to heal from a small perturbation increases linearly with the number of nodes in the system for sensitivity $\langle s\rangle \geq 1$, and sublinearly otherwise. The dashed line has slope 1 in this double-logarithmic plot. Each data point is the average over $t_{\text {heal }}$ for the subset of healing realizations. Realizations of network, initial condition and perturbation are the same as in Figure 2

$\langle s\rangle \approx 1$, small perturbations spread. Both for $\langle s\rangle \ll 1$ and $\langle s\rangle \gg 1$, the healing probability tends towards 1 . This effect is enhanced by increasing system size. In the limit of $n \rightarrow \infty$ one may expect a finite probability of non-healing only at $\langle s\rangle=1$. Then the dynamics is almost always stable under small perturbations.

The average time $t_{\text {heal }}$ to heal from small perturbations increases moderately with system size as shown in Figure 3, For average sensitivity above 1, we observe a linear increase $\left\langle t_{\text {heal }}\right\rangle \propto n$. For lower values of the average sensitivity, the increase is sublinear.

The dynamics we have studied so far is simple but not the only possibility to pass from the Boolean map to a continuous flow. In order to check to what extent our results depend on this choice we repeat simulations for $K=2$ with an alternative function $\tilde{f}$ (cf. Equation (2) now taking into account cooperative effects between inputs. Figure 4 shows that the same qualitative result obtains under this choice, see figure caption for details.

In summary, we have shown that the dynamics of large random networks of switch-like elements typically recovers from small perturbations of the state vector. Healing is observed naturally at low sensitivity. However, also large sensitivities of the nodes' functions render the longterm behaviour of the whole system insensitive to small perturbations. Instability is observed only in an intermediate sensitivity regime that shrinks as systems become larger.

The behaviour under small perturbations is essentially different from the established stability diagram for RBN. Under flip perturbations, RBN display a transition from 


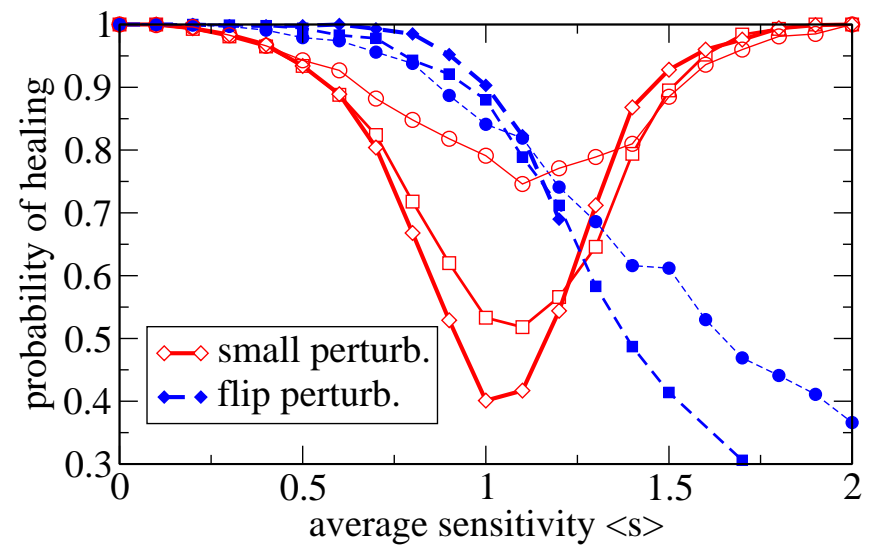

FIG. 4: (color online). Healing probabilities remain qualitatively the same (cf. Figure 2) when using the alternative transfer function $\tilde{f}_{i}(y)=\Theta\left(h_{i}(y)\right)$ with $h_{i}(y)=a y_{j} y_{k}+b_{1} y_{j}+$ $b_{2} y_{k}+c$; for node $i$ taking inputs from nodes $j$ and $k$. The parameters $a, b_{1}, b_{2}, c$ are chosen such that $h_{i}(y)=f_{i}(y)$ for $y_{j}, y_{k} \in\{0,1\}$. If, for instance, $f_{i}$ is an AND then $a=1$ and $b_{1}=b_{2}=c=0$ so $\tilde{f}_{i}(y)=1$ if and only if the product of inputs $y_{j} y_{k} \geq 1 / 2$. Each data point is the healing fraction of 1000 realizations of given average sensitivity and system size $n=30(\circ), 100(\square)$ and $300(\diamond)$. The perturbation amplitude $\epsilon_{i}$ is drawn from the uniform distribution on $[0 ; 0.01]$ independently for each node $i$. healing to non-healing (damage spreading) behaviour at average sensitivity 1 . It has been suggested that networks of regulatory switches position themselves at this transition [33], known as the edge of chaos [34]. Then some but not all flip perturbations spread and therefore allow for complex information processing without rendering the system unreliable under noise.

According to our findings, a complementary scenario is worth discussing. The apparent conflict between responsiveness to external input signals and resilience to intrinsic noise dissolves when these influences act as perturbations at separate scales: noise corresponds to small perturbations whilst input signals are interpreted as the flipping of a state. Under these assumptions, noise resilience and responsiveness are compatible rather than conflicting in the regime of average sensitivity above 1 . Systems that combine both beneficial properties are obtained "for free" in random networks of sufficiently sensitive switching elements.

Acknowledgments. - The authors thank Gunnar Boldhaus, Florian Greil and Thimo Rohlf for valuable comments. This work has been financially supported by VolkswagenStiftung through the initiative on Complex Networks as Phenomena across Disciplines.
[1] S. Bornholdt, Science 310, 449 (2005).

[2] H. de Jeong, J Comput Biol 9, 67 (2002).

[3] S. A. Kauffman, J Theor Biol 22, 437 (1969).

[4] I. Albert, J. Thakar, S. Li, R. Zhang, and R. Albert, Source Code Biol Med 3, 16 (2008).

[5] B. Drossel, Reviews of Nonlinear Dynamics and Complexity 1, 69 (2007).

[6] T. Helikar, J. Konvalina, J. Heidel, and J. A. Rogers, Proc Natl Acad Sci USA 105, 1913 (2008).

[7] D. Sahoo, J. Seita, D. Bhattacharya, M. A. Inlay, I. L. Weissman, S. K. Plevritis, and D. L. Dill, Proc Natl Acad Sci USA 107, 5732 (2010).

[8] H. A. Carteret, K. J. Rose, and S. A. Kauffman, Phys. Rev. Lett. 101, 218702 (2008).

[9] R. Albert and H. Othmer, J Theor Biol 223, 1 (2003).

[10] F. Li, T. Long, Y. Lu, Q. Ouyang, and C. Tang, Proc Natl Acad Sci USA 101, 4781 (2004).

[11] A. Samal and S. Jain, BMC Syst Biol 2, 21 (2008).

[12] M. I. Davidich and S. Bornholdt, PLoS ONE 3, e1672 (2008).

[13] M. Davidich and S. Bornholdt, J Theor Biol 255, 269 (2008).

[14] S. H. Strogatz, Nonlinear Dynamics and Chaos: With Applications to Physics, Biology, Chemistry and Engineering (Westview Press, Boulder, 1994).

[15] B. Derrida and Y. Pomeau, Europhys Lett 1, 45 (1986).

[16] S. Kauffman, C. Peterson, B. Samuelsson, and C. Troein, Proc Natl Acad Sci USA 100, 14796 (2003).

[17] I. Shmulevich, H. Lähdesmäki, E. R. Dougherty, J. Astola, and W. Zhang, Proc Natl Acad Sci USA 100, 10734 (2003).
[18] S. Kauffman, C. Peterson, B. Samuelsson, and C. Troein, Proc Natl Acad Sci USA 101, 17102 (2004).

[19] T. Rohlf, N. Gulbahce, and C. Teuscher, Phys Rev Lett 99, 248701 (2007).

[20] A. Pomerance, E. Ott, M. Girvan, and W. Losert, Proc Natl Acad Sci USA 106, 8209 (2009).

[21] T. P. Peixoto, Phys Rev Lett 104, 048701 (2010).

[22] A. Eldar and M. B. Eolwitz, Nature 467, 167 (2010).

[23] I. Lestas, G. Vinnicombe, and J. Paulsson, Nature 467, 174 (2010).

[24] L. Glass and S. Kauffman, J Theor Biol 34, 219 (1972).

[25] S. Braunewell and S. Bornholdt, J Theor Biol 258, 502 (2009).

[26] J. Norrell, B. Samuelsson, and J. Socolar, Phys Rev E 76, 46122 (2007).

[27] J. Norrell and J. Socolar, Phys Rev E 79, 61908 (2009).

[28] E. Gehrmann and B. Drossel, Phys Rev E 82, 046120 (2010).

[29] K. Klemm and S. Bornholdt, Proc Natl Acad Sci USA 102, 18414 (2005).

[30] K. Klemm and S. Bornholdt, Phys Rev E 72, 055101 (2005).

[31] S. Braunewell and S. Bornholdt, J Theor Biol 245, 638 (2007).

[32] I. Shmulevich and S. Kauffman, Phys Rev Lett 93, 48701 (2004).

[33] S. A. Kauffman, The Origins of Order (Oxford University Press, New York, 1993).

[34] C. G. Langton, Physica D 42, 12 (1990). 